\title{
The Stationary Distributions of Doubly Skew Ornstein-Uhlenbeck Processes and Markov-modulated Skew Ornstein-Uhlenbeck Processes
}

\author{
Danfeng Zhao ${ }^{1}$, Ye Liu ${ }^{2}$ \\ ${ }^{1,2}$ School of Sciences, Hebei University of Technology, Beichen district, Tianjin 300401, China
}

\begin{abstract}
In this paper, we consider the stationary density function of the doubly skew Ornstein-Uhlenbeck process. We present the explicit formula for the stationary density function and show that this process is positive Harris recurrent and geometrically ergodic. We expand our method to the more general cases in which the multiple parameters are present and we try to consider the stability of the skew Ornstein-Uhlenbeck process whose parameters depend on a finite-state and irreducible continuous-time Markov chain. Then we offer the stationary distribution equation of this bivariate process through their infinitesimal generator and the explicit stationary distributions are list as a special case.
\end{abstract}

Keywords: doubly skew Ornstein-Uhlenbeck process, markov-modulated process, stationary distribution.

\section{Introduction}

Skew diffusions have drawn attention in fields of financial engineering, physics, mathematical biology, etc. because of their potential applications, for example, [10], [13], [12], and [8]. The skew Brownian motion(abbr. skew BM) was first introduced by K. Ito and H. P. Mckean in[6], which is a simple but interesting diffusion process. The doubly skewed OU process is an extension of the skew Brownian motion. A general class of stochastic differential equations involving the local times of unknown process was first proved by Le Gall [5], and then developed by Barlow[11], Engelbert and Schmidt [4], Lejay [2], Bass and Chen [14] and many others.

We denote by $(\Omega, \mathrm{F}, P)$ a complete probability space and the filtration $\left\{F_{t}, t \geq 0\right\}$ is assumed to satisfy the usual conditions. For a process $X=\left\{X_{t}, t \geq 0\right\}$, the notation $\left(X_{t}, \mathrm{~F}_{t}\right)$ signifies that $X$ is adapted to the filtration $\left\{\mathrm{F}_{t}\right\}$. For parameters $\gamma, \sigma>0, b>a,|\xi 1|,|\xi 2|<1$, we define the doubly skewed Ornstein-Uhlenbeck (abbr. skew OU) process $\left(X_{t}, \mathrm{~F}_{t}\right)$ as a weak solution to

$d X_{t}=\gamma X_{t} d t+\sigma d B_{t}+\left(2 p_{1}-1\right) d \hat{L}_{t}^{X}(a)+\left(2 p_{2}-1\right) d \hat{L}_{t}^{X}(b)$,

where $B_{t}$ is a standard Brownian motion on some probability space $(\Omega, \mathrm{F}, P)$ and $\hat{L}_{t}^{X}(a)$ (resp. $\hat{L}_{t}^{X}(b)$ is the symmetric local time at $a$ (resp. $b$ ). It is well-known that for $p_{1}=0$ or $p_{2}=0$, the equation (1) is the skew OU process whose weak existence and pathwise uniqueness have been proved by S.Wang [16]. The aim of this paper is to elaborate the stationary of this kind of process.

When computing the stationary distribution, Karlin and
Taylor [15] offered a classical method which is not suitable for our process. We use the infinitesimal generator of doubly skewed OU process to derive the stationary density function. Then we expand our method to more general doubly skew OU process and the Markov-modulated skew OU process(abbr. MMSOU). There are a large amount of papers concentrating on Markov modulated process, because such a process can capture the switching of the market conditions. Many scholars investigate their applications in finance. For example, [3] and [7] studied how to price options.

The paper is organized as follows. Section 2 is concentrated on the explicit stationary density of doubly skew OU process, under the drift coefficient has only one parameter. Section 3 expands to the multiple-parameter case. Section 4 discusses the stability of the MMSOU, here we mainly consider the stability in distribution.

\section{Stationary distribution for the doubly skewed process}

A doubly skewed OU process which starts from any initial point in its state space behaves like the ordinary OU process until it reaches the skew point $a$ (resp. $b$ ), causing upwards with probability $p_{1}$ (resp. $p_{2}$ ) and downwards with probability $1-p_{1}$ (resp. $1-p_{2}$ ). In this section, we will derive the stationary density function of the doubly skewed OU process $\left\{X_{t}\right\}$ defined in (1). Before giving the theorem about the stationary density of process $\left\{X_{t}\right\}$, we first introduce some basic definitions.

Definition 2.1. The Markov process $\Phi$ is called ergodic if an invariant probability $\pi$ exists and

$\lim _{t \rightarrow \infty}\left\|P^{t}(y, \cdot)-\pi\right\|=0, y \in Y$,

and is called geometrically ergodic if for some $B<\infty, \beta<1$, 


\section{International Journal of Science and Research (IJSR) \\ ISSN (Online): 2319-7064}

Index Copernicus Value (2013): 6.14 | Impact Factor (2015): 6.391

$\left\|P^{t}(y, \cdot)-\pi\right\|_{f} \leq B f(\mathrm{x}) \beta^{t}, y \in Y, t>0$,

with $f(\cdot)=|\cdot|^{2}+1$.

Theorem 2.2. The doubly skew OU process $\left\{X_{t}\right\}$ defined in (1) is positive Harris recurrent and $V$-uniform ergodic.

Proof. Let $C$ be the set of functions $f \in C_{b}(R)$, $f^{\prime}, f^{\prime \prime} \in C_{b}(R) \backslash\{a, b\}$ and $f^{\prime}$ satisfies $p_{1} f^{\prime}(a+)=\left(1-p_{1}\right) f^{\prime}(a-)$, $p_{2} f^{\prime}(b+)=\left(1-p_{2}\right) f^{\prime}(b-)$, at points $a$ and $b$. Recall that $I=(0, \infty)$. For $f \in C$, applying the generalized Ito formula to $f\left(X_{t}\right)$, we have

$$
\begin{aligned}
d f\left(X_{s}\right)= & f^{\prime}\left(X_{s}\right) d X_{s}+\frac{1}{2} f^{\prime \prime}\left(X_{s}\right) d\langle X\rangle_{s}+\frac{1}{2}\left[f^{\prime}(a+)-f^{\prime}(a-)\right] d \hat{L}_{s}^{X}(a) \\
& +\frac{1}{2}\left[f^{\prime}(b+)-f^{\prime}(b-)\right] d \hat{L}_{S}^{X}(b) \\
= & -\gamma X_{s} f^{\prime}\left(X_{s}\right) d s+\sigma f^{\prime}\left(X_{s}\right) d B_{s}+\xi_{1} f^{\prime}\left(X_{s}\right) d \hat{L}_{s}^{X}(a) \\
& +\xi_{2} f^{\prime}\left(X_{s}\right) d \hat{L}_{S}^{X}(b)+\frac{1}{2} f^{\prime \prime}\left(X_{s}\right) \sigma^{2} d s \\
& +\frac{1}{2}\left[f^{\prime}(a+)-f^{\prime}(a-)\right] d \hat{L}_{s}^{X}(a)+\frac{1}{2}\left[f^{\prime}(b+)-f^{\prime}(b-)\right] d \hat{L}_{S}^{X}(b) \\
= & -\gamma X_{s} f^{\prime}\left(X_{s}\right) d s+\sigma f^{\prime}\left(X_{s}\right) d B_{s}+\frac{1}{2} f^{\prime \prime}\left(X_{s}\right) \sigma^{2} d s,
\end{aligned}
$$

where the last equity holds because of the fact

$$
\begin{aligned}
& \left(2 p_{1}-1\right) f^{\prime}\left(X_{s}\right) d \hat{L}_{S}^{X}(a)+\left(2 p_{2}-1\right) f^{\prime}\left(X_{s}\right) d \hat{L}_{S}^{X}(b) \\
& +\frac{1}{2}\left[f^{\prime}(a+)-f^{\prime}(a-)\right] d \hat{L}_{S}^{X}(a)+\frac{1}{2}\left[f^{\prime}(b+)-f^{\prime}(b-)\right] d \hat{L}_{S}^{X}(b) \\
= & \frac{2 p_{1}-1}{2}\left[f^{\prime}(a+)-f^{\prime}(a-)\right] d \hat{L}_{S}^{X}(a)+\frac{2 p_{2}-1}{2}\left[f^{\prime}(b+)-f^{\prime}(b-)\right] d \hat{L}_{S}^{X}(b) \\
& +\frac{1}{2}\left[f^{\prime}(a+)-f^{\prime}(a-)\right] d \hat{L}_{S}^{X}(a)+\frac{1}{2}\left[f^{\prime}(b+)-f^{\prime}(b-)\right] d \hat{L}_{S}^{X}(b)
\end{aligned}
$$$$
=0 \text {. }
$$

Then

$f\left(X_{t}\right)=f\left(X_{0}\right)+\int_{0}^{t}\left[-\gamma X_{s} f^{\prime}\left(X_{s}\right) d s+\frac{1}{2} f^{\prime \prime}\left(X_{s}\right) \sigma^{2} d s\right]+\int_{0}^{t} \sigma f^{\prime}\left(X_{s}\right) d B_{s}$. Fo $\mathrm{r}$ any $f \in C$, the infinitesimal generator of $\{X t, t \geq 0\}$ is defined by

$$
\begin{aligned}
\operatorname{A} f(x) & =\lim _{t \rightarrow \infty} \frac{E_{x} f\left(X_{t}\right)-E_{x} f\left(X_{0}\right)}{t} \\
& =\frac{1}{2} \sigma^{2} f^{\prime \prime}(x)-\gamma x f^{\prime}(x), f \in C .
\end{aligned}
$$

Define

$$
V(x)= \begin{cases}p_{1} p_{2}(x-a), & x<a, \\ \left(1-p_{1}\right) p_{2}(x-a), & a \leq x<b, \\ \left(1-p_{1}\right)\left(1-p_{2}\right)(x-b), & x \geq b .\end{cases}
$$

It is easy to check $V(x) \in C$ is norm-like, i. e. $V(x) \rightarrow \infty$ as $x \rightarrow \infty$. Thus, by operating the generator $\mathrm{A}_{n}$ on $V$, we get

$$
\mathrm{A}_{n} V(x)= \begin{cases}\gamma p_{1} p_{2} x, & x<a, \\ \gamma\left(1-p_{1}\right) p_{2} x, & a \leq x<b, \\ \gamma\left(1-p_{1}\right)\left(1-p_{2}\right) x, & x \geq b .\end{cases}
$$

After a simple algebraic calculation shows that

$$
\mathrm{A}_{n} V(x)+2 \gamma V(x)= \begin{cases}\gamma p_{1} p_{2} x-2 \gamma p_{1} p_{2} a, & x<a, \\ \gamma\left(1-p_{1}\right) p_{2} x-2 \gamma\left(1-p_{1}\right) p_{2} a, & a \leq x<b, \\ \gamma\left(1-p_{1}\right)\left(1-p_{2}\right) x-2 \gamma\left(1-p_{1}\right)\left(1-p_{2}\right) b, & x \geq b,\end{cases}
$$
and let $\theta_{1}$ to be the maximum of $\mathrm{A}_{n} V(x)+2 \gamma V(x), \quad x \in R$, then

$\mathrm{A}_{n} V(x) \leq-2 \gamma V(x)+\theta_{1}$.

Now, the following is obvious

$\mathrm{A}_{n} V(x) \leq-c(x+1)+\theta_{2}$,

where $c=\min \left\{2 \gamma p_{1} p_{2}, 2 \gamma\left(1-p_{1}\right) p_{2}, 2 \gamma\left(1-p_{1}\right)\left(1-p_{2}\right)\right\}$ and $\theta_{2}$ is the maximum of $\mathrm{A}_{n} V(x)+2 \gamma V(x)$. Next, comparing the equation(2) and (3), we derive that $\theta^{*}=\max \left\{\theta_{1}, \theta_{2}\right\}$.

It proves that the process $\left\{X_{t}, t \geq 0\right\}$ is positive Harris recurrent from the formula (3) and the Theorem 4.4 in [9], and an V-uniformly ergodic from formula (2), Corollary 4.9 and Theorem 4.12 in [1].

Theorem 2.3. The doubly skewed OU process $X_{t}$ defined in (1) is positive Harris recurrent and geometrically ergodic, and the density of the unique stationary distribution for $X_{t}$ is as follows:

$$
q(x)= \begin{cases}\frac{\left(1-p_{1}\right)\left(1-p_{2}\right)}{\Lambda} e^{-\frac{\gamma x^{2}}{\sigma^{2}}}, & -\infty<x<a, \\ \frac{p_{1}\left(1-p_{2}\right)}{\Lambda} e^{-\frac{\gamma x^{2}}{\sigma^{2}}}, & a<x<b, \\ \frac{p_{1} p_{2}}{\Lambda} e^{-\frac{\gamma x^{2}}{\sigma^{2}}}, & x>b,\end{cases}
$$

where

$$
\begin{aligned}
\Lambda= & \left(1-p_{1}\right)\left(1-p_{2}\right) \int_{-\infty}^{a} e^{-\frac{\gamma x^{2}}{\sigma^{2}}} d x \\
& +p_{1}\left(1-p_{2}\right) \int_{-\infty}^{a} e^{-\frac{\gamma x^{2}}{\sigma^{2}}} d x \\
& +p_{1} p_{2} \int_{-\infty}^{a} e^{-\frac{\gamma x^{2}}{\sigma^{2}}} d x .
\end{aligned}
$$

Proof. If $q(x)$ is the stationary density of the doubly skew OU process, then the following equation is satisfied

$$
\begin{aligned}
& \int_{-\infty}^{\infty} f(x)\left[\frac{\sigma^{2}}{2} q^{\prime \prime}(x)+\gamma q(x)+\gamma x q^{\prime}(x)\right] d x \\
+ & \frac{\sigma^{2}}{2}\left[q(a-) f^{\prime}(a-)-q(a+) f^{\prime}(a+)\right]+\frac{\sigma^{2}}{2}\left[q(b-) f^{\prime}(b-)-q(b+) f^{\prime}(b+)\right] \\
+ & f(a)\left[(-\gamma) a q(a-)+\gamma a q(a+)+\frac{\sigma^{2}}{2} q^{\prime}(a+)-\frac{\sigma^{2}}{2} q^{\prime}(a-)\right] \\
+ & f(b)\left[(-\gamma) b q(b-)+\gamma b q(b+)+\frac{\sigma^{2}}{2} q^{\prime}(b+)-\frac{\sigma^{2}}{2} q^{\prime}(b-)\right] .
\end{aligned}
$$

For the arbitrary of $f \in C$, it can be deduced that

$$
\left\{\begin{array}{l}
\frac{\sigma^{2}}{2} q^{\prime \prime}(x)+\gamma q(x)+\gamma x q^{\prime}(x)=0, \\
q(a-) f^{\prime}(a-)=q(a+) f^{\prime}(a+), \\
q(b-) f^{\prime}(b-)=q(b+) f^{\prime}(b+) .
\end{array}\right.
$$

Finally, considering the condition $\int_{-\infty}^{\infty} q(x) d x=1$, we get the

\section{Volume 5 Issue 6, June 2016 www.ijsr.net}




\section{International Journal of Science and Research (IJSR) \\ ISSN (Online): 2319-7064}

Index Copernicus Value (2013): 6.14 | Impact Factor (2015): 6.391

unique stationary density $q(x)$ given by (4).

\section{Extension to multiple-parameter case}

The method can be extended to the more general cases in which the multiple parameters are presented. We consider the following general doubly skew OU process:

$d X_{t}=\left(\alpha-\gamma X_{t}\right) d t+\sigma d B_{t}+\left(2 p_{1}-1\right) d \hat{L}_{t}^{X}(a)+\left(2 p_{2}-1\right) d \hat{L}_{t}^{X}(b)$,

where $\hat{L}_{t}^{X}(a)$ and $\hat{L}_{t}^{X}(b)$ are the local time at $a$ and $b$, respectively. In the above model, our goal is to derive the stationary density of joint parameters $\alpha$ and $\gamma$.

Theorem 3.1. The doubly skewed OU process $X_{t}$ defined in (5) is positive Harris recurrent and geometrically ergodic, and the density of the unique stationary distribution for $X_{t}$ is as follows:

$$
q(x)= \begin{cases}\frac{\left(1-p_{1}\right)\left(1-p_{2}\right)}{\Lambda} e^{-\frac{\gamma x^{2}}{\sigma^{2}}}\left(x-\frac{\alpha}{\gamma}\right)^{2}, & -\infty<x<a, \\ \frac{p_{1}\left(1-p_{2}\right)}{\Lambda} e^{-\frac{\gamma x^{2}}{\sigma^{2}}}\left(x-\frac{\alpha}{\gamma}\right)^{2}, & a<x<b, \\ \frac{p_{1} p_{2}}{\Lambda} e^{-\frac{\gamma x^{2}}{\sigma^{2}}}\left(x-\frac{\alpha}{\gamma}\right)^{2}, & x>b,\end{cases}
$$

where

$$
\begin{aligned}
\Lambda= & \left(1-p_{1}\right)\left(1-p_{2}\right) \int_{-\infty}^{a} e^{-\frac{\gamma x^{2}}{\sigma^{2}}}\left(x-\frac{\alpha}{\gamma}\right)^{2} d x \\
& +p_{1}\left(1-p_{2}\right) \int_{-\infty}^{a} e^{-\frac{\gamma x^{2}}{\sigma^{2}}}\left(x-\frac{\alpha}{\gamma}\right)^{2} d x \\
& +p_{1} p_{2} \int_{-\infty}^{a} e^{-\frac{\gamma x^{2}}{\sigma^{2}}}\left(x-\frac{\alpha}{\gamma}\right)^{2} d x .
\end{aligned}
$$

Proof. Let $C$ be the set of functions $f \in C_{b}(R)$, $f^{\prime}, f^{\prime \prime} \in C_{b}(R) \backslash\{a, b\}$ and $f^{\prime}$ satisfies $p_{1} f^{\prime}(a+)=\left(1-p_{1}\right) f^{\prime}(a-)$, $p_{2} f^{\prime}(b+)=\left(1-p_{2}\right) f^{\prime}(b-)$, at points $a$ and $b$. Recall that $I=(0, \infty)$. For $f \in C$, applying the generalized Ito formula to $f\left(X_{t}\right)$, we have

$$
\begin{aligned}
d f\left(X_{s}\right)= & f^{\prime}\left(X_{s}\right) d X_{s}+\frac{1}{2} f^{\prime \prime}\left(X_{s}\right) d\langle X\rangle_{s}+\frac{1}{2}\left[f^{\prime}(a+)-f^{\prime}(a-)\right] d \hat{L}_{s}^{X}(a) \\
& +\frac{1}{2}\left[f^{\prime}(b+)-f^{\prime}(b-)\right] d \hat{L}_{S}^{X}(b) \\
= & \left(\alpha-\gamma X_{s}\right) f^{\prime}\left(X_{s}\right) d s+\sigma f^{\prime}\left(X_{s}\right) d B_{s}+\xi_{1} f^{\prime}\left(X_{s}\right) d \hat{L}_{s}^{X}(a) \\
& +\xi_{2} f^{\prime}\left(X_{s}\right) d \hat{L}_{s}^{X}(b)+\frac{1}{2} f^{\prime \prime}\left(X_{s}\right) \sigma^{2} d s \\
& +\frac{1}{2}\left[f^{\prime}(a+)-f^{\prime}(a-)\right] d \hat{L}_{s}^{X}(a)+\frac{1}{2}\left[f^{\prime}(b+)-f^{\prime}(b-)\right] d \hat{L}_{S}^{X}(b) \\
= & \left(\alpha-\gamma X_{s}\right) f^{\prime}\left(X_{s}\right) d s+\sigma f^{\prime}\left(X_{s}\right) d B_{s}+\frac{1}{2} f^{\prime \prime}\left(X_{s}\right) \sigma^{2} d s,
\end{aligned}
$$

then

$$
f\left(X_{t}\right)=f\left(X_{0}\right)+\int_{0}^{t}\left[\left(\alpha-\gamma X_{s}\right) f^{\prime}\left(X_{s}\right) d s+\frac{1}{2} f^{\prime \prime}\left(X_{s}\right) \sigma^{2} d s\right] d s+\int_{0}^{t} \sigma f^{\prime}\left(X_{s}\right) d B_{s} .
$$

For any $f \in C$, the infinitesimal generator of $\{X t, t \geq 0\}$ is defined by

$$
\begin{aligned}
\operatorname{A} f(x) & =\lim _{t \rightarrow \infty} \frac{E_{x} f\left(X_{t}\right)-E_{x} f\left(X_{0}\right)}{t} \\
& =\frac{1}{2} \sigma^{2} f^{\prime \prime}(x)+(\alpha-\gamma x) f^{\prime}(x), f \in C .
\end{aligned}
$$

Then by employing the methods of integration by parts twice, the above equation becomes

$$
\int_{-\infty}^{\infty} f(x)\left[\frac{\sigma^{2}}{2} q^{\prime \prime}(x)+\gamma q(x)-(\alpha-\gamma x) q^{\prime}(x)\right] d x
$$

$+\frac{\sigma^{2}}{2}\left[q(a-) f^{\prime}(a-)-q(a+) f^{\prime}(a+)\right]+\frac{\sigma^{2}}{2}\left[q(b-) f^{\prime}(b-)-q(b+) f^{\prime}(b+)\right]$

$+f(a)\left[(\alpha-\gamma a) q(a-)-(\alpha-\gamma a) q(a+)+\frac{\sigma^{2}}{2} q^{\prime}(a+)-\frac{\sigma^{2}}{2} q^{\prime}(a-)\right]$

$+f(b)\left[(\alpha-\gamma b) q(b-)-(\alpha-\gamma b) q(b+)+\frac{\sigma^{2}}{2} q^{\prime}(b+)-\frac{\sigma^{2}}{2} q^{\prime}(b-)\right]$.

Now it follows that

$\left\{\begin{array}{l}\frac{\sigma^{2}}{2} q^{\prime \prime}(x)+\gamma q(x)-(\alpha-\gamma x) q^{\prime}(x)=0, \\ q(a-) f^{\prime}(a-)=q(a+) f^{\prime}(a+), \\ q(b-) f^{\prime}(b-)=q(b+) f^{\prime}(b+) .\end{array}\right.$

Therefore, the equation (6) can be obtained.

\section{Extension to the Markov-modulated skew OU process case}

In this section, we consider the stability of a skew OU process $\left\{X_{t}, t \geq 0\right\}$,whose parameters depend on a finite-state and irreducible continuous time Markov chain $\left\{J_{t}, t \geq 0\right\}$. We first define the MMSOU $\left\{X_{t}, J_{t}\right\}, t \geq 0$ on the state space $R \times E \quad$, where $E=\{1,2, \ldots, N\}$ for some finite integer $N$.Suppose that $(\Omega, \mathrm{F}, P)$ is a filtered probability space with the filtration $\left\{F_{t}: t \geq 0\right\}$ satisfying the usual condition. The first component is also called observable process, $X t$, which satisfies the following stochastic differential equations (abbr. SDE) with local time

$d X_{t}=\left(\alpha-\gamma_{J t} X_{t}\right) d t+\sigma_{J t} d B_{t}+(2 p-1) d \hat{L}_{t}^{X}(a), a>0, \alpha \in(0,1)$, where $B_{t}$ is a standard Brownian motion and $\hat{L}_{t}^{x}(a)$ is the symmetric local time at $a$. The second component of the bivariate process, $J_{t}$, is an irreducible right-continuous Markov chain taking values in $E=\{1,2, \ldots, N\}$ defined on the same probability space. In addition, assume that $J t$ and $B t$ are independent. For $J t=i, i \in E, \gamma_{i}, \sigma_{i}>0$ are some constants and $\hat{L}_{t}^{X}(a)$ is the symmetric local time. The Markov chain $J t$ is characterized by its generator $V=\left(v_{i j}\right)_{N \times N}$ satisfying

$W=\left\{J_{t+\Delta}=j \mid J_{t}=i\right\}= \begin{cases}v_{i j} \Delta+o(\Delta), & \text { if } i \neq j, \\ 1+v_{i j} \Delta+o(\Delta), & \text { if } i \neq j,\end{cases}$

Where $\Delta>0$ and $v_{i j}>0$ is the transition rate from $i$ to $j$ if $i \neq j$, $v_{i i}=-\sum_{j \neq i} v_{i j}<0$.

Let $\pi=\left(\pi_{1}, \pi_{2}, \ldots, \pi_{N}\right)$ be the stationary distribution of $J t$. Because we have assumed that $J t$ is an irreducible 


\section{International Journal of Science and Research (IJSR) \\ ISSN (Online): 2319-7064}

Index Copernicus Value (2013): 6.14 | Impact Factor (2015): 6.391

continuous-time Markov chain, it is well known that $\pi$ is the unique solution to

$$
\pi V=0, \sum_{i=1}^{N} \pi_{i}=1 .
$$

Next, we will introduce the infinitesimal generator of the MMSOU $\left\{\left(X_{t}, J_{t}\right)\right\}, t \geq 0$. Let $C$ be the set of functions $f(x, i)$ on $R \times E$ that are bounded and continuous in $x$ for which the first and second derivatives are bounded and continuous for all $x$ except at the skew point $a$ with $p f^{\prime}(a+, i)=(1-p) f^{\prime}(a-, i)$ and $f^{\prime \prime}(a+, i)=f^{\prime \prime}(a-, i)$ for $i \in E$ where

$$
\begin{gathered}
f^{\prime}(x, i)=\frac{\partial f}{\partial x}(x, i), f^{\prime \prime}(x, i)=\frac{\partial^{2} f}{\partial^{2} x} f(x, i) \\
f^{\prime}(a, i)=\frac{1}{2}\left[f^{\prime}(a+, i)+f^{\prime}(a-, i)\right] .
\end{gathered}
$$

Define

$$
E_{(x, i)}=\lim _{t \rightarrow 0} \frac{1}{t}\left[E_{(x, i)} f\left(X_{t}, J_{t}\right)-E_{(x, i)} f\left(X_{0}, J_{0}\right)\right] .
$$

The infinitesimal generator of the Markov process $\left\{\left(X_{t}, J_{t}\right)\right\}$ $t \geq 0$ is defined as follows

$(\mathrm{A} f)(x, i)=\lim _{t \rightarrow 0} \frac{1}{t}\left[E_{x, i} f\left(X_{t}, J_{t}\right)-E_{x} f\left(X_{0}, J_{0}\right)\right]$.

For any $f \in C$, using the Ito Tanaka formula, we obtain

$$
\begin{aligned}
& E_{(x, i)} f\left(X_{t}, J_{t}\right)-E_{(x, i)} f\left(X_{0}, J_{0}\right) \\
= & E_{(x, i)} f\left(X_{t}, J_{t}\right)-E_{(x, i)} f\left(X_{t}, J_{0}\right)+E_{(x, i)} f\left(X_{t}, J_{0}\right)-E_{(x, i)} f\left(X_{0}, J_{0}\right) \\
= & \left(1+v_{i i} t\right) E_{(x, i)} f\left(X_{t}, i\right)+\sum_{j \neq i} v_{i j} E_{(x, i)} f\left(X_{t,} j\right)-E_{(x, i)} f\left(X_{t}, i\right) \\
& +E_{(x, i)}\left[\int_{0}^{t} f^{\prime}\left(X_{s}, J_{0}\right)\left(-\gamma_{J_{s}}\right) X_{s} d s+\frac{1}{2} \int_{0}^{t} f^{\prime \prime}\left(X_{s}, J_{0}\right) \sigma_{J_{s}}^{2} d s\right] \\
& +\int_{0}^{t} f^{\prime}\left(X_{s}, J_{0}\right)(2 p-1) d \hat{L}_{s}^{X}(a)+\frac{1}{2}\left[f^{\prime}\left(a+, J_{0}\right)-f^{\prime}\left(a-, J_{0}\right)\right] \hat{L}_{t}^{X}(a) \\
= & E_{(x, i)}\left[\int_{0}^{t} f^{\prime}\left(X_{s}, J_{0}\right)\left(-\gamma_{J_{s}}\right) X_{s} d s+\frac{1}{2} \int_{0}^{t} f^{\prime \prime}\left(X_{s}, J_{0}\right) \sigma_{J_{s}}^{2} d s\right] \\
& +\sum_{j \in E} v_{i j} E_{(x, i)} f\left(X_{t, j}\right) .
\end{aligned}
$$

Then it is easy to see that the infinitesimal generator of the process $\left(X_{t}, J_{t}\right), t \geq 0$ satisfies

$$
(\mathrm{A} f)(x, i)=\sum_{j \in E} v_{i j} f\left(X_{t, j}\right)-\gamma_{i} x f^{\prime}(x, i)+\frac{1}{2} \sigma_{i}^{2} f^{\prime \prime}(x, i) \text {, }
$$

where $f^{\prime}(x, i)$ and $f^{\prime \prime}(x, i)$ are the first and second order partial derivatives at $x$ and the domain of A includes the set $C$.

Proposition 4.1. Let $\left(X_{\infty}, J_{\infty}\right)$ be the stationary distribution of process $\left(X_{t}, J_{t}\right)$. Then the stationary densities $q_{i}(x)$ are the solutions of the following equation

$$
A(x) q^{\prime \prime}(x)+B(x) q^{\prime}(x)+C q(x)+V^{T} q(x)=0,
$$

satisfying the following conditions

$p q(a-)=(1-p) a(a+)$

$\int_{-\infty}^{\infty} q(x) d x=\pi^{T}$

$B(a)(q(a+)-q(a-))+A\left(q^{\prime}(a+)-q^{\prime}(a-)\right)=0$,

where $V^{T}$ is the transpose of $V$ and

$$
\begin{aligned}
& A=\operatorname{diag}\left(\frac{1}{2} \sigma_{1}^{2}, \ldots, \sigma_{N}^{2}\right), \\
& B(X)=\operatorname{diag}\left(\gamma_{1} x, \ldots, \gamma_{N} x\right), \\
& C=\operatorname{diag}\left(\gamma_{1}, \ldots, \gamma_{N}\right), \\
& q(x)=\left[q_{1}(x), \ldots, q_{N}(x)\right]^{T},
\end{aligned}
$$

with the definition diag

$$
\left(a_{1}, a_{2}, \ldots, a_{n}\right)=\left(\begin{array}{cccc}
a_{1} & 0 & 0 & 0 \\
0 & a_{2} & 0 & 0 \\
0 & 0 & \ldots & 0 \\
0 & 0 & 0 & a_{n}
\end{array}\right) .
$$

Generally speaking, it is difficult to calculate the closed form of (8) and (9). While in some special cases, the closed solutions of stationary densities can be figured out.

Proof: From exercise 57, P.394 in [15], we know that a probability density $q_{i}(x)$ is a stationary density for $X t$ if and only if the following equation is satisfied

$$
\begin{aligned}
& \sum_{x \in E} \int_{-\infty}^{\infty}(\mathrm{A} f)(x, i) q_{i}(x) d x \\
= & \sum_{i \in E} \int_{-\infty}^{\infty}\left[\frac{1}{2} \sigma_{i}^{2} f^{\prime \prime}(x, i)-\gamma_{i} x f^{\prime}(x, i)+\sum_{j \in E} q_{i j} f(x, j)\right] q_{i}(x) d x,
\end{aligned}
$$

and $p f^{\prime}(a+)=(1-p) f^{\prime}(a-)$, we obtain that

$$
\begin{aligned}
& \sum_{i \in E} \int_{-\infty}^{\infty}(\mathrm{A} f)(x, i) q_{i}(x) d x \\
= & \sum_{i \in E} \int_{-\infty}^{\infty} f(x, i)\left[\frac{1}{2} \sigma_{i}^{2} q^{\prime \prime}(x, i)+\gamma_{i} x q^{\prime}(x, i)+\gamma_{i} q_{i}(x)+\sum_{j \in E} q_{i j} q_{j}(x)\right] d x \\
& +\sum_{i \in E} \frac{1}{2} \sigma_{i}^{2}\left[q_{i}(a-) f^{\prime}(a-, i)-q_{i}(a+) f^{\prime}(a+, i)\right] \\
& +\sum_{i \in E} f(a, i)\left[\gamma_{i} a q_{i}(a-)+\gamma_{i} a q_{i}(a+)+\frac{1}{2} \sigma_{i}^{2} q_{i}^{\prime}(a+)-\frac{1}{2} \sigma_{i}^{2} q_{i}^{\prime}(a-)\right] .
\end{aligned}
$$

Since for any $f \in C,(8),(9),(10)$ and (11) are obtained.

\section{Special Cases}

example. Set $N=2, a=0$ and the generator of the Markov chain $J$ to be

$$
Q=\left(\begin{array}{cc}
-w & w \\
v & -v
\end{array}\right),
$$

where $w, v>0$.From (7), we obtain $\mathrm{w} \pi_{1}=\mathrm{v} \pi_{2}$ and $\pi_{1}=\frac{v}{w+v}$, We also assume $\sigma_{i}=\sqrt{\gamma_{i}}$, for $i=1,2 ;$ with these assumptions, the closed form of stationary densities for $(X t, J t)$ can be obtained:

$q_{1}(x)= \begin{cases}\frac{2(1-p)}{\sqrt{\pi}} \pi_{1} e^{-x 2}, & x<0, \\ \frac{2 p}{\sqrt{\pi}} \pi_{1} e^{-x 2}, & x \geq 0,\end{cases}$
$q_{2}(x)= \begin{cases}\frac{2(1-p)}{\sqrt{\pi}} \pi_{2} e^{-x^{2}}, & x<0, \\ \frac{2 p}{\sqrt{\pi}} \pi_{2} e^{-x^{2}}, & x \geq 0,\end{cases}$

Proof. We have done above, from Proposition 4.1, the

\section{Volume 5 Issue 6, June 2016 www.ijsr.net}




\section{International Journal of Science and Research (IJSR) \\ ISSN (Online): 2319-7064}

Index Copernicus Value (2013): 6.14 $\mid$ Impact Factor (2015): 6.391

stationary densities $q_{1}(x)$ and $q_{2}(x)$ should satisfy the following equation group

$\left\{\begin{array}{l}\frac{1}{2} \gamma_{1} q^{\prime \prime}(x)+\gamma_{1} x q^{\prime \prime}(x)+\gamma_{1} q_{1}(x)-w q_{1}(x)+v q_{2}(x)=0, \\ \frac{1}{2} \gamma_{1} q^{\prime \prime}(x)+\gamma_{1} x q^{\prime \prime}(x)+\gamma_{1} q_{1}(x)-w q_{1}(x)+v q_{2}(x)=0,\end{array}\right.$

and the following conditions should be satisfied

$$
\left\{\begin{array}{l}
q_{1}(0)=q_{2}(0)=0, \\
w q_{1}(a-)=(1-w) q_{1}(a+), w q_{2}(a-)=(1-w) q_{2}(a+), \\
\int_{-\infty}^{\infty} q_{1}(x) d x=\pi_{1}, J_{-\infty}^{\infty} q_{2}(x) d x=\pi_{2}, \\
\gamma_{1} a\left(q_{1}(a+)-q_{1}(a-)\right)+\frac{1}{2} \gamma_{1}\left(q_{1}^{\prime}(a+)-q_{1}^{\prime}(a-)\right)=0, \\
\gamma_{2} a\left(q_{2}(a+)-q_{2}(a-)\right)+\frac{1}{2} \gamma_{2}\left(q_{2}^{\prime}(a+)-q_{2}^{\prime}(a-)\right)=0,
\end{array}\right.
$$

Adding the two equations in (14), we obtain

$\frac{1}{2}\left(\gamma_{1} q_{1}^{\prime \prime}(x)+\gamma_{2} q_{2}^{\prime \prime}(x)\right)+x\left(\gamma_{1} q_{1}^{\prime}+\gamma_{2} q_{2}^{\prime}(x)\right)+\gamma_{1} q_{1}(x)+\gamma_{2} q_{2}(x)=0$.

Assume that $h(x)=\gamma_{1} q_{1}(x)+\gamma_{2} q_{2}(x)$, then $h(x)$ should satisfy the following equation

$$
\frac{1}{2} h^{\prime \prime}(x)+x h^{\prime}(x)+h(x)=0 .
$$

Through simple calculation, the general solution of above equation should be $h(x)=c e^{-2 x}$ with $c$ is a constant. Because of the conditions for $q_{1}(x)$ and $q_{2}(x), h(x)$ must satisfy the following conditions

$$
\left\{\begin{array}{l}
h(0)=\gamma_{1} q_{1}(0)+\gamma_{2} q_{2}(0)=0, \\
h(a-)=\gamma_{1} q_{1}(a-)+\gamma_{2} q_{2}(a-)=\frac{1-\alpha}{\alpha}\left[\gamma_{1} q_{1}(a+)+\gamma_{2} q_{2}(a+)\right]=\frac{1-\alpha}{\alpha} h(a+), \\
\int_{-\infty}^{\infty} h(x) d x=\int_{-\infty}^{\infty}\left[\gamma_{1} q_{1}(x)+\gamma_{2} q_{2}(x)\right] d x=\gamma_{1} \pi_{1}+\gamma_{2} \pi_{2} .
\end{array}\right.
$$

Combining the above conditions and the general solution of $h(x)$, we get

$$
\begin{aligned}
h(x) & =\gamma_{1} q_{1}(x)+\gamma_{2} q_{2}(x), \\
& = \begin{cases}\frac{2(1-p)}{\sqrt{\pi}}\left[\pi_{1} \gamma_{1}+\pi_{2} \gamma_{2}\right] e^{-x 2}, & x<0, \\
\frac{2 p}{\sqrt{\pi}}\left[\pi_{1} \gamma_{1}+\pi_{2} \gamma_{2}\right] e^{-x 2}, & x \geq 0,\end{cases}
\end{aligned}
$$

and the solutions of $q_{1}(x)$ and $q_{2}(x)$ are exhibited in (12) and (13).

\section{References}

[1] A.Budhiraja, and C.Lee, "Long time asymptotics for constrained diffusions in polyhedral domains," Stochasticastic Processes Their Applications, 117(8), 1014-1036, 2007.

[2] A.Lejay, " On the decomposition of excursions measures of processes whose generators have diffusion coefficients discontinuous at one point," Markov Processes and Related Fields, 8, 117-126, 2002.

[3] A.Jobert, L.C.G.Rogers, "Option pricing with Markovmodulated dynamics," SIAM Journal on Control and Optimization, 44, 2063-2078, 2006.
[4] H.J.Engelbert, W.Schmidt, "Strong Markov continuous local martingales and solution of one-dimensional stochastic differential equations (Part III)," Mathematische Nachrichten, 151, 149-197, 1991.

[5] J.F.Le Gall, "Temps locaux et equations differentielles stochastiques,"Springer, Berlin, 1983.

[6] K.It` o, H.P.McKean, "Diffusion Processes and Their Sample Paths,"Springer Berlin, 37(4), 1060-1062, 2001.

[7] L.Bo, Y.Wang, X.Yang, "Markov-modulated jumpdiffusions for currency option pricing," Insurance Mathematics and Economics, 46, 461-469, 2010.

[8] M.Barlow, K.Burdzy, H.Kaspi, and A.Mandelbaum, "Coalescence of skew Brownian motions," Electronic Communications in Probability., 5, 57-66, 2001.

[9] Meyn, P.Sean, RL.Tweedie, "Stability of Markovian processes III: FosterCLyapunov criteria for continuous-time processes," Addvanced in Applied Probability, 25(3), 518C548, 2010.

[10] M.I.Freidlin, A.D.Wentzell, "Diffusion processes on graphs and the averging principle," Annals of Probability, 21, 2215-2245, 1993.

[11] M.T. Barlow, "Skew Brownian motion and a one dimensional stochastic differential equation," Stochastics, 25, 1-2, 1998.

[12] N.Portenko, "A probabilistic representation for the solution of one problem of mathematical physics," Ukrainian Mathematical Journal, 52, 1457-1469, 2000.

[13] R.Lang, "Effective conductivity and skew Brownian motion," J Stat Phys, 80, 125-146, 1995.

[14] R.F.Bass, Z.Q.Chen, "One-dimensional stochastic differential equations with singular and degenerate coefficients," Sankhya: The Indian Journal of Statistics, 19-45, 2005.

[15] S.Karlin, H.M.Taylor, "A second course in stochastic processes," Academic Press, New Work, 1981.

[16] S.Wang, S.Song, Y.Wang, "Skew Ornstein-Uhlenbeck processes and their finacial applications," Journal of Computational and Applied Mathematics, 273, 363-382, 2015. 